\title{
Structures, Operations and their Applications to Topology
}

\author{
Geetha Jeyalakshmi $\mathbf{R}^{\mathrm{a}}$ and Dass $\mathrm{K}^{\mathrm{b}}$ \\ Research Scholar, Department of Mathematics, The M.D.T Hindu College, \\ Affiliated to Manonmaniam Sundaranar University, Tirunelveli -India \\ ${ }^{b}$ Department of Mathematics, The M.D.T. Hindu College,Tirunelveli-627010, India.
}

Article History: Received: 11 January 2021; Accepted: 27 February 2021; Published online: 5 April 2021

\begin{abstract}
A structure on a non empty set $\mathrm{X}$ is a collection of subsets of $\mathrm{X}$. Any kind of topology on a non empty set $\mathrm{X}$ is a special structure on $\mathrm{X}$. A filter and a filter base on $\mathrm{X}$ are examples of structures. Also any ideal of subsets of $\mathrm{X}$ is a structure. In this paper several structures are classified and the binary relations and operations on structures are discussed. Furthermore structures on a topological space are also discussed.
\end{abstract}

Keywords: Structure, hyper relation, hyper union, hyper intersection, micro relation

\section{Introduction}

A structure on a non empty set $\mathrm{X}$ is a collection of subsets of $\mathrm{X}$. Any kind of topology on a non empty set $\mathrm{X}$ is a special structure on X. A filter and a filterbase in X are examples of a structure. Also any ideal (Jankovic \& Hamlet, 1990) of subsets of $X$ is a structure. In this paper several structures are classified and the binary relations and operations on structures are discussed. In particular several structures on a topological space and their common properties are discussed. The second section deals with the preliminaries that are needed for the paper. The notions of hyper intersction and hyper union of structures have been introduced and investigated in Section3. The hyper difference operator on structures has been introduced and studied in the fourth section and the fifth section deals with the application of the above operators to the structures induced by a topology.

\section{Preliminaries}

In this paper certain basic concepts and results in topology are given. Let A and B be the subsets of a topological space $(\mathrm{X}, \tau)$. The Interior and Closure operators on $\mathrm{A}$ are respectively denoted by $\mathrm{Int} \mathrm{A}$ and $\mathrm{Cl} \mathrm{A}$. The following expressions will be useful in sequel.

Correspondind Author: Geetha jeyalakshmi

email: geetha1010@gmail.com

\section{Citation Information:}

Structures, Operations and Their Applications to Topology

\subsection{Expression}

Int $\mathrm{A} \subseteq$ Int $C l$ Int $\mathrm{A} \subseteq C l$ Int $\mathrm{A} \subseteq C l$ Int $C l \mathrm{~A} \subseteq C l \mathrm{~A}$.

\subsection{Expression}

Int $\mathrm{A} \subseteq$ Int $C l$ Int $\mathrm{A} \subseteq \operatorname{Int} C l \mathrm{~A} \subseteq C l$ Int $C l \mathrm{~A} \subseteq C l \mathrm{~A}$.

\subsection{Definition}

A is called

(i).b-open(Andrijevic,1996) in (X, $\tau)$ if $\mathrm{A} \subseteq C l$ Int $\mathrm{A} \cup \operatorname{Int} C l \mathrm{~A}$ and b-closed if $C l \operatorname{Int} \mathrm{A} \cap \operatorname{Int} C l \mathrm{~A} \subseteq \mathrm{A}$,

(ii). *b-open(Indira et.al.,2012) if $\mathrm{A} \subseteq C l$ Int $\mathrm{A} \cap \operatorname{Int} C l \mathrm{~A}$ and *b-closed if $C l$ Int $\mathrm{A} \cup \operatorname{Int} C l \mathrm{~A} \subseteq \mathrm{A}$,

(iii). $\mathrm{b}^{\#-o p e n(U s h a ~ P a r a m e s w a r i ~ e t . a l ., 2014) ~ i f ~} \mathrm{~A}=C l$ Int $\mathrm{A} \cup \operatorname{Int} C l \mathrm{~A}$ and $\mathrm{b}^{\#}$-closed if Cl Int $\mathrm{A} \cap$ Int $C l \mathrm{~A}=\mathrm{A}$.

Let $X$ be a set. By a structure on $X$ we wean a collection of subsets of $X$. For example if $X=\{a, b, c\}$ then the subsets $\{a\},\{b\}$ and $\{a, c\}$ of $X$ constitute a structure of $X$, denoted by $[\{a\},\{b\},\{a, c\}]$. Throughout this paper $\mathrm{P}, \mathrm{Q}, \mathrm{R}, \mathrm{S}, \Omega$ are structures on $\mathrm{X}$.

2.4. Definitions

(i) $2^{\mathrm{X}}$ denotes the whole structure on $\mathrm{X}$.

(ii) If $A$ is a subset of $X$ then the structure $[A]$ is known as a singleton structure of $X$.

(iii) $[\mathrm{X}]$ denotes the absolute structure of .

(iv) $\Phi=$ the empty structure or the null structure on $X$.

(v) If $\mathrm{A}$ and $\mathrm{B}$ are two distinct subsets of $\mathrm{X}$ then $[\mathrm{A}, \mathrm{B}]=[\mathrm{B}, \mathrm{A}]$ is a doublton structure of $\mathrm{X}$. 
Generally structures can be compared by the set inclusion relations namely $\subseteq, \supseteq, \subset$ and $\supset$. The hyper relations namely $\subset, \supset$ and micro relations $\Subset, \ni$ on structures have been already discussed in (Jeyalakshmi et.al., 2021). It has been established that the relations $\odot$ and $\supset$ are both transitive and reflexive.

\subsection{Definition}

(i) If $\mathrm{P} \subseteq \mathrm{Q}$ then $\mathrm{P}$ is a substructure of $\mathrm{Q}$ and $\mathrm{Q}$ is a superstructure of $\mathrm{P}$.

(ii) $\mathrm{P}$ is a hyper substructure of $\mathrm{Q}$ denoted by $\mathrm{P} \subset \mathrm{Q}$ if for all $\mathrm{A} \in \mathrm{P}$ there exists $\mathrm{B} \in \mathrm{Q}$ with $\mathrm{A} \subseteq \mathrm{B}$.

(iii) $\mathrm{P}$ is a hyper superstructure of $\mathrm{Q}$ denoted by $\mathrm{P} \supset \mathrm{Q}$ if for all $\mathrm{A} \in \mathrm{P}$ there is a $\mathrm{B} \in \mathrm{Q}$ with $\mathrm{A} \supseteq \mathrm{B}$.

(iv) $\mathrm{P}$ is a micro substructure of $\mathrm{Q}$ denoted by $\mathrm{P} \Subset \mathrm{Q}$ if $\mathrm{A} \in \mathrm{P} \Rightarrow \mathrm{A} \subseteq \mathrm{B}$ for every $\mathrm{B} \in \mathrm{Q}$.

(v) $\mathrm{P}$ is a micro superstructure of $\mathrm{Q}$ denoted by $\mathrm{P} \supset \mathrm{Q}$ if $\mathrm{A} \in \mathrm{P} \Rightarrow \mathrm{A} \supseteq \mathrm{B}$ for every $\mathrm{B} \in \mathrm{Q}$.

A topology of $\mathrm{X}$ induces several structures on $\mathrm{X}$. The following are structures induced by a topology.

\subsection{Examples}

(i) $\mathrm{bO}(\mathrm{X}, \tau)$ - structure of b-open sets and $\mathrm{bC}(\mathrm{X}, \tau)$ - structure of b-closed sets.

(ii) $* \mathrm{bO}(\mathrm{X}, \tau)$ - structure of $* \mathrm{~b}$-open sets and $* \mathrm{bC}(\mathrm{X}, \tau)$-structure of $* \mathrm{~b}$-closed sets.

(iii) $b^{\#} O(X, \tau)$-structure of $b^{\#}$-open sets and $b^{\#} C(X, \tau)$-structure of $b^{\#}$ - closed sets.

The following hyper inclusion diagrams always hold for any topological space $(X, \tau)$.

\subsection{Diagram}

(i) $* b O(X, \tau) \subset b O(X, \tau)$ and $b^{\#} \mathrm{O}(\mathrm{X}, \tau) \subset \mathrm{bO}(\mathrm{X}, \tau)$.

(ii) ${ }^{*} \mathrm{bC}(\mathrm{X}, \tau) \subset \mathrm{bC}(\mathrm{X}, \tau)$ and $\mathrm{b}^{\#} \mathrm{C}(\mathrm{X}, \tau) \subset \mathrm{bC}(\mathrm{X}, \tau)$.

\section{Hyper Intersection and Hyper Union} section

The concepts of hyper intersection and hyper union operators have been introduced and discussed in this

\subsection{Definition}

$\mathrm{P} \cap \mathrm{Q}=\{\mathrm{A} \cap \mathrm{B}: \mathrm{A} \in \mathrm{P}$ and $\mathrm{B} \in \mathrm{Q}\}=$ The hyper intersection of $\mathrm{P}$ with $\mathrm{Q}$.

$\mathrm{P} \uplus \mathrm{Q}=\{\mathrm{A} \cup \mathrm{B}: \mathrm{A} \in \mathrm{P}$ and $\mathrm{B} \in \mathrm{Q}\}=$ The hyper union of $\mathrm{P}$ with $\mathrm{Q}$.

\subsection{Example}

Let $X=\{a, b, c\}, P=[\{a, b\},\{a, c\},\{b, c\}]$ and $Q=[\{a\},\{b\},\{c\},\{a, b\},\{a, c\}], P \cap Q=[\{a, b\},\{a, c\}], P \cap Q=[\varnothing$, $\{a\},\{b\},\{c\},\{a, b\},\{a, c\}], P \cup Q=[\{a\},\{b\},\{c\},\{a, b\},\{a, c\},\{b, c\}]$,

$\mathrm{P} \uplus \mathrm{Q}=[\{\mathrm{a}, \mathrm{b}\},\{\mathrm{a}, \mathrm{c}\},\{\mathrm{b}, \mathrm{c}\}, \mathrm{X}]$.

\subsection{Proposition}

(i) $\mathrm{P} \biguplus \bar{Q}=\mathrm{P}$ and $\mathrm{P} \cap \Phi=\Phi$.

(ii) $\mathrm{P} \biguplus[\varnothing]=\mathrm{P}$ and $\mathrm{P} \cap[\varnothing]=[\varnothing]$.

(iii) $\mathrm{P} \uplus[\mathrm{X}]=[\mathrm{X}]$ and $\mathrm{P} \cap[\mathrm{X}]=\mathrm{P}$.

(iv) $\mathrm{P} \uplus 2^{\mathrm{X}}=2^{\mathrm{X}}$ and $\mathrm{P} \subseteq \mathrm{P} \cap 2^{\mathrm{X}} \subseteq 2^{\mathrm{X}}$.

\subsection{Proposition}

(i) $\mathrm{P} \subseteq \mathrm{P} \uplus \mathrm{P}$ and $\mathrm{P} \subseteq \mathrm{P} \cap \mathrm{P}$.

(ii) $\mathrm{P} \cap \mathrm{P} \subset \mathrm{P} \subset \mathrm{P} \uplus \mathrm{P}$.

(iii) $\mathrm{P} \uplus \mathrm{Q}=\mathrm{Q} \uplus \mathrm{P}$ and $\mathrm{P} \cap \mathrm{Q}=\mathrm{Q} \cap \mathrm{P}$.

(iv) $\mathrm{P} \uplus(\mathrm{Q} \uplus \mathrm{R})=(\mathrm{P} \uplus \mathrm{Q}) \biguplus \mathrm{R}$ and $\mathrm{P} \cap(\mathrm{Q} \cap \mathrm{R})=(\mathrm{P} \cap \mathrm{Q}) \cap \mathrm{R}$.

(v) $\mathrm{P} \cap(\mathrm{Q} \uplus \mathrm{R}) \subseteq(\mathrm{P} \cap \mathrm{Q}) \biguplus(\mathrm{P} \cap \mathrm{R})$ and $\mathrm{P} \uplus(\mathrm{Q} \cap \mathrm{R}) \subseteq(\mathrm{P} \uplus \mathrm{Q}) \cap(\mathrm{P} \uplus \mathrm{R})$.

\subsection{Proposition}

If $\mathrm{P}=[\mathrm{A}]$ then $\mathrm{P} \cap \mathrm{P}=\mathrm{P}=\mathrm{P} \uplus \mathrm{P}$ and if $\mathrm{P}=[\mathrm{A}, \mathrm{B}]$ where $\mathrm{A} \neq \mathrm{B}$ and $\mathrm{A} \subseteq \mathrm{B}$ then $\mathrm{P} \cap \mathrm{P}=\mathrm{P}=\mathrm{P} \uplus \mathrm{P}$.

Geetha Jeyalakshmi and Dass

\subsection{Definition}

$\mathrm{P}$ is a nested structure if for any two members $\mathrm{A}, \mathrm{B}$ of $\mathrm{P}$ either $\mathrm{A} \subseteq \mathrm{B}$ or $\mathrm{A} \supseteq \mathrm{B}$ holds.

\subsection{Proposition}

If $\mathrm{P}$ is a nested structure then $\mathrm{P} \cap \mathrm{P}=\mathrm{P}=\mathrm{P} \uplus \mathrm{P}$.

\subsection{Proposition}



(i) If $P \Subset Q$ and $R \Subset S$ and $P ค R \Subset Q ค S$ and $P \uplus R \Subset Q \uplus S$.
(ii) If $P \subset Q$ and $R \subset S$ and $P \cap R \subset Q \cap S$ and $P \uplus R \subset Q \uplus S$.
3.9. Proposition
(i) If $P \ni Q$ and $R \ni S$ and $P \cap R \ni Q \cap S$ and $P \uplus R \ni Q \uplus S$.
(ii) If $P \ni Q$ and $R \ni S$ and $P \cap R \ni Q \cap S$ and $P \uplus R \ni Q \uplus S$.

\section{Hyper Difference Operator}

\subsection{Definition}

$\mathrm{P} \ominus \mathrm{Q}=[\mathrm{A} \backslash \mathrm{B}: \mathrm{A} \in \mathrm{P}$ and $\mathrm{B} \in \mathrm{Q}]=$ The hyper difference of $\mathrm{Q}$ from $\mathrm{P}$.

\subsection{Proposition}

(i) $\mathrm{P} \ominus[\varnothing]=\mathrm{P}$ and $[\varnothing] \ominus \mathrm{P}=[\varnothing]$.

(ii) $\mathrm{P} \ominus[\mathrm{X}]=[\varnothing]$ and $[\mathrm{X}] \ominus \mathrm{P}=[\mathrm{X} \backslash \mathrm{A}: \mathrm{A} \in \mathrm{P}]$.

(iii) $\mathrm{P} \subseteq \mathrm{P} \Theta 2^{\mathrm{X}}$ and $[\mathrm{X}] \Theta \mathrm{P} \subseteq 2^{\mathrm{X}} \Theta \mathrm{P}$.

(iv) $[\mathrm{A}] \Theta[\mathrm{A}]=[\varnothing]$ and $\mathrm{P} \Theta \mathrm{P} \neq[\varnothing]$ if $\mathrm{P}$ contains more than one mmeber.

\subsection{Proposition}

(i) $\mathrm{P} \ominus \mathrm{Q} \subseteq \mathrm{P} \Theta(\mathrm{P} \cap \mathrm{Q})$.

(ii) If $\mathrm{P} \cap \mathrm{Q}=[\varnothing]$ then $\mathrm{P} \ominus \mathrm{Q}=\mathrm{P}$.

(iii) If $\mathrm{P} \ominus \mathrm{Q}=[\varnothing]$ then $\mathrm{P} \Subset \mathrm{Q}$ and $\mathrm{P} \subset \mathrm{Q}$.

\subsection{Proposition}

(i) $(\mathrm{P} \ominus \mathrm{Q}) \ominus \mathrm{R}=\mathrm{P} \ominus(\mathrm{Q} \uplus \mathrm{R}) \subseteq(\mathrm{P} \ominus \mathrm{R}) \Theta(\mathrm{Q} \ominus \mathrm{R})$.

(ii) $\mathrm{P} \ominus(\mathrm{Q} \ominus \mathrm{R}) \subseteq(\mathrm{P} \ominus \mathrm{Q}) \uplus(\mathrm{P} \cap \mathrm{R})$.

(iii) $(\mathrm{P} \uplus \mathrm{Q}) \ominus \mathrm{R} \subseteq(\mathrm{P} \ominus \mathrm{R}) \uplus(\mathrm{Q} \ominus \mathrm{R})$.

(iv) $(\mathrm{P} \cap \mathrm{Q}) \Theta \mathrm{R} \subseteq(\mathrm{P} \ominus \mathrm{R}) \cap(\mathrm{Q} \ominus \mathrm{R})$

(v) $\mathrm{P} \cap(\mathrm{Q} \ominus \mathrm{R}) \subseteq(\mathrm{P} \cap \mathrm{Q}) \Theta(\mathrm{P} \cap \mathrm{R})$.

(vi) $\mathrm{P} \uplus(\mathrm{Q} \ominus \mathrm{R}) \subseteq(\mathrm{P} \ominus \mathrm{Q}) \uplus(\mathrm{P} \ominus \mathrm{Q}) \uplus(\mathrm{P} \cap \mathrm{Q} \cap \mathrm{R})$.

(vii) $\mathrm{P} \Theta(\mathrm{Q} \uplus \mathrm{R}) \subseteq(\mathrm{P} \ominus \mathrm{Q}) \curvearrowright(\mathrm{P} \ominus \mathrm{R})$

(viii) $\mathrm{P} \ominus(\mathrm{Q} \cap \mathrm{R}) \subseteq(\mathrm{P} \ominus \mathrm{Q}) \uplus(\mathrm{P} \ominus \mathrm{R})$.

\subsection{Proposition}

(i) $\mathrm{P} \cap\left(2^{\mathrm{X}} \ominus \mathrm{P}\right) \neq[\varnothing]$ if $\mathrm{P} \neq[\varnothing]$ and $\mathrm{P} \uplus\left(2^{\mathrm{X}} \ominus \mathrm{P}\right) \neq 2^{\mathrm{X}}$.

(ii) $2^{\mathrm{X}} \Theta\left(2^{\mathrm{X}} \ominus \mathrm{P}\right) \neq \mathrm{P}$ and $\mathrm{X} \ominus(\mathrm{X} \ominus \mathrm{P})=\mathrm{P}$

(iii) $\mathrm{P} \cap([\mathrm{X}] \ominus \mathrm{P})=\mathrm{P} \ominus \mathrm{P}$ and $\mathrm{P} \uplus([\mathrm{X}] \ominus \mathrm{P})=[\mathrm{X}] \Theta(\mathrm{P} \ominus \mathrm{P})$

\subsection{Proposition}

(i) $\mathrm{P} \cap([\mathrm{X}] \ominus \mathrm{Q})=\mathrm{P} \ominus \mathrm{Q}$ and $\mathrm{P} \uplus([\mathrm{X}] \ominus \mathrm{Q})=[\mathrm{X}] \Theta(\mathrm{Q} \ominus \mathrm{P})$.

(ii) $\mathrm{P} \cap \mathrm{Q}=[\varnothing] \Rightarrow \mathrm{P} \Subset[\mathrm{X}] \ominus \mathrm{Q}$.

(iii) $[\mathrm{X}] \Theta(\mathrm{Q} \uplus \mathrm{R})=([\mathrm{X}] \ominus \mathrm{Q}) \cap([\mathrm{X}] \Theta \mathrm{R})$.

(iv) $[\mathrm{X}] \Theta(\mathrm{Q} \cap \mathrm{R})=([\mathrm{X}] \ominus \mathrm{Q}) \uplus([\mathrm{X}] \ominus \mathrm{R})$

\subsection{Remark}

The results (iii) and (iv) of the above proposition are also valid for arbitrary hyper union and arbitrary hyper intersection of structures as given below.

\subsection{Proposition}

(i) $[\mathrm{X}] \ominus\left(\uplus\left\{\mathrm{P}_{\alpha}: \alpha \in \mathrm{J}\right\}\right)=\cap\left\{[\mathrm{X}] \ominus \mathrm{P}_{\alpha}: \alpha \in \mathrm{J}\right\}$

(ii) $[\mathrm{X}] \ominus\left(\cap\left\{\mathrm{P}_{\alpha}: \alpha \in \mathrm{J}\right\}\right)=\uplus\left\{[\mathrm{X}] \ominus \mathrm{P}_{\alpha}: \alpha \in \mathrm{J}\right\}$

\subsection{Proposition}


(ii) If $\mathrm{P} \ni \mathrm{Q}$ then $[\mathrm{X}] \ominus \mathrm{Q} \ni[\mathrm{X}] \ominus \mathrm{P}$

\section{Applicatuons to Topology}

Let $\mathrm{T}$ denote the collection of all topologies on a non empty set $\mathrm{X}$.

\subsection{Lemma}

(i) $\mathrm{P} \cap \mathrm{Q}=\cup[\mathrm{P} \cap[\mathrm{B}]: \mathrm{B} \in \mathrm{Q}]$

(ii) $\mathrm{P} \uplus \mathrm{Q}=\cup[\mathrm{P} \uplus[\mathrm{B}]: \mathrm{B} \in \mathrm{Q}]$

\subsection{Proposition}

Let $\mathrm{P} \in \mathrm{T}, \mathrm{Q} \in \mathrm{T}$.

(i) $\mathrm{P} \cap[\varnothing, \mathrm{X}]=\mathrm{P}$

(ii) $\mathrm{P}$ is a hyper substructure of $[\varnothing, \mathrm{X}]$

(iii) $\mathrm{P} \uplus[\varnothing, \mathrm{X}]=\mathrm{P}$.

(iv) $\mathrm{P} \cap 2^{\mathrm{X}}=2^{\mathrm{X}}$

(iii) $\mathrm{P} \cap \mathrm{Q}=\mathrm{Q} \cap \mathrm{P}$ will be a basis for some topology on $\mathrm{X}$

(iv) $\mathrm{P} \uplus \mathrm{Q}$ is a generalized topology $($ Csaszar,20002) on $\mathrm{X}$

\subsection{Proposition}

Let $\mathrm{P} \in \mathrm{T}$.

(i) $\mathrm{P} \cap \mathrm{P}=\mathrm{P}=\mathrm{P} \uplus \mathrm{P}$

(ii) $\mathrm{P} \ominus \mathrm{P}=\mathrm{P} \cap \mathrm{P}^{\prime}$ where $\mathrm{P}^{\prime}$ is the collection of closed sets with respect to $\mathrm{P}$.

Let $(\mathrm{X}, \tau)$ be a topological space and $\Omega$ be a collection of subsets of X. Let $C l \Omega=[C l \mathrm{~A}: \mathrm{A} \in \Omega]$ and Int $\Omega$ $=[\operatorname{Int} \mathrm{A}: \mathrm{A} \in \Omega]$.

\subsection{Proposition}

(i) $\mathrm{bO}(\mathrm{X}, \tau) \subset C l$ Int $\mathrm{bO}(\mathrm{X}, \tau) \uplus \operatorname{Int} \mathrm{Cl} \mathrm{bO}(\mathrm{X}, \tau)$.

(ii) $\mathrm{bC}(\mathrm{X}, \tau) \ni C l$ Int $\mathrm{bC}(\mathrm{X}, \tau) ค \operatorname{Int} C l \mathrm{bC}(\mathrm{X}, \tau)$.

(iii) $C l \mathrm{bO}(\mathrm{X}, \tau)=C l$ Int $C l \mathrm{bO}(\mathrm{X}, \tau)$.

(iv) Int $\mathrm{bC}(\mathrm{X}, \tau)=\operatorname{Int} C l$ Int $\mathrm{bC}(\mathrm{X}, \tau)$.

\subsection{Proposition}

(i) $* \mathrm{bO}(\mathrm{X}, \tau) \subset C l$ Int $* \mathrm{bO}(\mathrm{X}, \tau) ค \operatorname{Int} C l * \mathrm{bO}(\mathrm{X}, \tau)$.

(ii) $* \mathrm{bC}(\mathrm{X}, \tau) \unlhd C l$ Int $* \mathrm{bC}(\mathrm{X}, \tau) \uplus \operatorname{Int} C l * \mathrm{bC}(\mathrm{X}, \tau)$.

(iii) $C l * \mathrm{bO}(\mathrm{X}, \tau)=C l$ Int $* \mathrm{bO}(\mathrm{X}, \tau)=. C l$ Int $C l * \mathrm{bO}(\mathrm{X}, \tau)$.

(iv) Int $* \mathrm{bC}(\mathrm{X}, \tau)=\operatorname{IntCl} * \mathrm{bC}(\mathrm{X}, \tau)=\operatorname{IntCl} \operatorname{Int} * \mathrm{bC}(\mathrm{X}, \tau)$.

\subsection{Proposition}

(i) $\mathrm{b}^{\sharp} \mathrm{O}(\mathrm{X}, \tau) \subset C l$ Int $\mathrm{b}^{\sharp} \mathrm{O}(\mathrm{X}, \tau) \uplus \operatorname{Int} C l \mathrm{~b}^{\#} \mathrm{O}(\mathrm{X}, \tau)$

(ii) $\mathrm{b}^{\#} \mathrm{C}(\mathrm{X}, \tau) \subset C l \operatorname{Int} \mathrm{b}^{\#} \mathrm{C}(\mathrm{X}, \tau) ค \operatorname{Int} C l \mathrm{~b}^{\#} \mathrm{C}(\mathrm{X}, \tau)$.

\subsection{Proposition}

Let $(\mathrm{X}, \tau)$ be a topological space .

(i) Int $C l\left(2^{\mathrm{x}} ค 2^{\mathrm{X}}\right) \subset \operatorname{Int} \mathrm{Cl}\left(2^{\mathrm{X}}\right) ค \operatorname{Int} C l\left(2^{\mathrm{X}}\right)$

(ii) $C l$ Int $\left(2^{\mathrm{X}} ค 2^{\mathrm{X}}\right) \subset C l$ Int $\left(2^{\mathrm{X}}\right) ค C$ Cl Int $\left(2^{\mathrm{X}}\right)$

(iii) Int $C l\left(2^{\mathrm{x}}\right) \uplus \operatorname{Int} C l\left(2^{\mathrm{x}}\right) \subset \operatorname{Int} C l\left(2^{\mathrm{x}} \uplus 2^{\mathrm{X}}\right)$

(iv) $C l$ Int $\left(2^{\mathrm{X}}\right) \uplus C l$ Int $\left(2^{\mathrm{X}}\right) \subset C l \operatorname{Int}\left(2^{\mathrm{x}} \uplus 2^{\mathrm{X}}\right)$

(v) Cl Int $C l\left(2^{\mathrm{X}}\right) \uplus C l \operatorname{Int} C l\left(2^{\mathrm{X}}\right)=C l$ Int $C l\left(2^{\mathrm{X}} \uplus 2^{\mathrm{X}}\right)$ 
(vi) Int Cl Int $\left(2^{\mathrm{X}}\right) \cap \operatorname{Int} C l$ Int $\left(2^{\mathrm{X}}\right)=\operatorname{Int} C l \operatorname{Int}\left(2^{\mathrm{X}} \cap 2^{\mathrm{X}}\right)$

Structures, Operations and Their Applications to Topology

\section{References}

1. Andrijevic, D., (1996). On b-open sets. Mat. Vesnik, 48(pp.59-64).

2. Csaszar, A.,(2002). Generalized topology, Generalized continuity. Acta.Math.Hungar, 96(4)

3. (pp. 351-357).

4. Geetha Jeyalakshmi, R., Dass, K.,(2021, Feb.24-25). Structures and Relations, International

5. Conference on Advanced Research in Mathematical Sciences(ICARMS-2021), India.(Online).

6. Geetha Jeyalakshmi, R., Dass, K., Pasunkilipandian, S., (2018, Dec.05-11). On Strucutres induced

7. by a bitopology, International Workshop and Conference on Topology and Applications

8. (IWCTA 2018). Kerela, India.

9. Indira, T, Rekha, K., (2012). Applications of *b-open sets and **b-open sets in topological

10. spaces, Annals of Pure and Applied Mathematics 1(1) (pp. 44-56).

11. Jankovic, D., Hamlet ,T. R., (1990). New topologies from old via ideals, Amer.Math.Monthly, 97

12. (pp. 295-310).

13. Usha Parameswari, R., Thangavelu, P., (2014). On $\quad \mathrm{b}^{\#}$-open sets, InternationalJournal of

14. Mathematics Trends and Technology, 3(5) (pp.202-218). 